\title{
Performance Optimization of Microstrip Antenna Array Using Frequency Selective Surfaces
}

\author{
Valdez A. Almeida Filho ${ }^{1}$, Antonio Luiz P. S. Campos ${ }^{2}$, \\ ${ }^{1}$ Graduate Program in Electrical and Computational Engineering \\ Federal University of Rio Grande do Norte (UFRN) \\ Av. Senador Salgado Filho, 3000, Natal, RN, Brazil, CEP: 59072-970 \\ Tel.: +55-21-84-3215-3767, e-mail: valdez.filho@ gmail.com \\ ${ }^{2}$ Communication Engineering Department \\ Federal University of Rio Grande do Norte (UFRN) \\ Av. Senador Salgado Filho, 3000, Natal, RN, Brazil, CEP: 59072-970 \\ Tel./fax: +55-21-84-3342-2516, e-mail: antonio.luiz@pq.cnpq.br
}

\begin{abstract}
This paper presents the application of frequency selective surfaces in antenna arrays as an alternative to improve radiation parameters of the array. A microstrip antenna array between two FSS was proposed for application in WLAN and LTE 4G systems. Several parameters have been significantly improved, in particular the bandwidth, gain and radiation efficiency, compared with a conventional array. Numerical and measured results are presented.
\end{abstract}

Index Terms - FSS, LTE, Microstrip Antena Array, WLAN.

\section{INTRODUCTION}

The development of telecommunications in recent years has given rise to a variety of services that use a specific frequency band for operation. Some of these services are designed to access the Internet via wireless networks, such as WLAN (Wireless Local Area Network), UMTS (Universal Mobile Telecommunications System) and LTE (Long Term Evolution) through mobile terminals, such as laptops or cell phones.

WLAN based networks (2400 to $2483.5 \mathrm{MHz}$ ) had a very rapid expansion due to numerous advantages such as the absence of wires between users and access points, which provide greater flexibility in the reception area, allowing the access to many locations, making it possible to create adhoc networks without prior planning, and facilitating the establishment of these networks.

In the field of mobile telephony we have, as a first example, the service of cellular, or WWAN (Wireless Wide Area Network), comprising the sub-band UMTS (1920 to $2170 \mathrm{MHz}$ ), which is part of the third generation technology, and LTE (2500 to $2690 \mathrm{MHz}$ ), which is part of the fourth generation technology, which was developed with the goal of bringing to cell phones, video, image and a higher speed access to the Internet.

Since each service operates at specific frequency bands, and in some cases with polarization and different radiation characteristics, it is necessary to integrate different antennas at the devices. To avoid the use of several antennas to operate the services, one possible solution is to use compact multi-band or broadband antennas. 
These antennas must have low cost, minimum weight, low profile and they must be able to maintain good performance over the frequency bands for which they were designed to operate. This technological trend has focused much on the design of microstrip antennas. With a simple geometry, the microstrip antennas offer many advantages that normally do not appear in other type of antennas. In contrast, the microstrip antenna offers the drawback of displaying a narrow bandwidth and low gain, low directivity, and low radiation efficiency to the simplest forms, which restricts their use [1], [2].

When some radiation parameters are not achieved with a single radiating element, we can use a technique that consists in associating radiating elements in a geometric and electrical configuration. This new antenna formed by multiple elements is the microstrip antenna array. In addition to increasing the bandwidth, with an array we can obtain higher levels of gain and directivity compared with a microstrip antenna [3], [4].

The microstrip antenna arrays have many applications in the area of Telecommunications. Among the latest applications found in literature, we can mention the use of arrays for satellite communications [5], [6], applications in L-band networks for GNSS/GPS [7] in wireless sensor networks [8] and textile substrates for communications centered on the human body [9]. A microstrip array using multilevel elements based on fractal geometry was proposed to obtain high-directivity, to reduction the size of the array, fractal geometry was used, thus reducing the complexity of the feeding network and the overall array [10].

Although microstrip arrays can be used in several applications. One of the problems of using arrays is related to the occupied space, since arrays have, for the most part, higher dimensions compared with single antennas. Another frequent problem is related to gain and directivity. To obtain higher levels of gain and directivity we must put more elements in the array. This leads to an increase in the dimensions of the structure.

One way to solve arrays' problems is the application of frequency selective surfaces (FSS) to the antenna array. Such interaction has the purpose of obtaining desired radiation characteristics for the antenna array from changes in their radiation parameters, such as bandwidth, gain and directivity. Another method to overcome the limitations of arrays is use of optimization techniques to design a single element antenna having similar performances as arrays, but with a lower patch area [11].

Frequency selective surfaces have been applied to antenna or antenna array in order to increase bandwidth in [12], [13]. The authors used an FSS EBG structure, it had characteristic bands insertion of operating/rejection in a given structure, achieving an increase in bandwidth of a specific antenna or antenna array. The increase in parameters such as gain and directivity using FSS applied to antennas and antenna arrays have been reported in [14], [15]. We can also mention the use of FSS to reduce the radar cross section (RCS) [16]. Other applications of FSS in microstrip antenna arrays found in literature are in multiband and high-directivity microstrip patch antenna arrays [17], [18].

This work has been proposed to improve the performance of the main radiation parameters of 
microstrip antenna arrays (bandwidth, gain, directivity, front-to-back ratio and reduced surface waves) through the use of frequency selective surfaces (FSS) coupled with the array. To realize this study, we designed two arrays of microstrip antennas. A conventional array and one with truncated ground plane, in order to increase the bandwidth and miniaturize the elements of the array. In the second array we applied two FSS to obtain better radiation parameters. The results show that the new array integrated with the FSS has better gain, radiation efficiency, bandwidth and reduced surface waves, when compared to the arrays without FSS.

\section{PROPOSED ARRAY}

The design of the proposed array starts with a design of a conventional array, narrow band, used to operate in WLAN frequency band (centered at $2.45 \mathrm{GHz}$ ). The geometry used was a rectangular patch with inset fed, as shown in Fig. 1. The inset fed is used to improve the impedance matching between the element and the feed line.

The dimensions of the patch are: $\mathrm{W}=37.34 \mathrm{~mm}, \mathrm{~L}=29.09 \mathrm{~mm}$, $\mathrm{y} 0=6.19 \mathrm{~mm}$ e $\mathrm{L} 0=14.52 \mathrm{~mm}$. The feed is obtained through a microstrip line, since it facilitates the integration of the array with other RF circuits. The substrate used was the fiberglass (FR4) with $\varepsilon_{\mathrm{r}}=4.4$ and $h=1.6 \mathrm{~mm}$, due to their low cost and facility to buy it. For the present study we designed an array with two elements. Fig. 2 illustrates the geometry of the array with all dimensions used and the discontinuities in feed network.

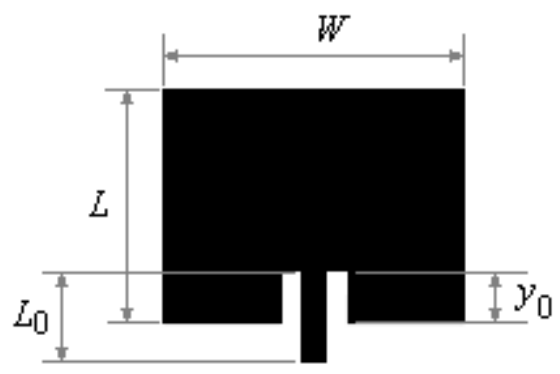

Fig. 1. Patch geometry used in the array.

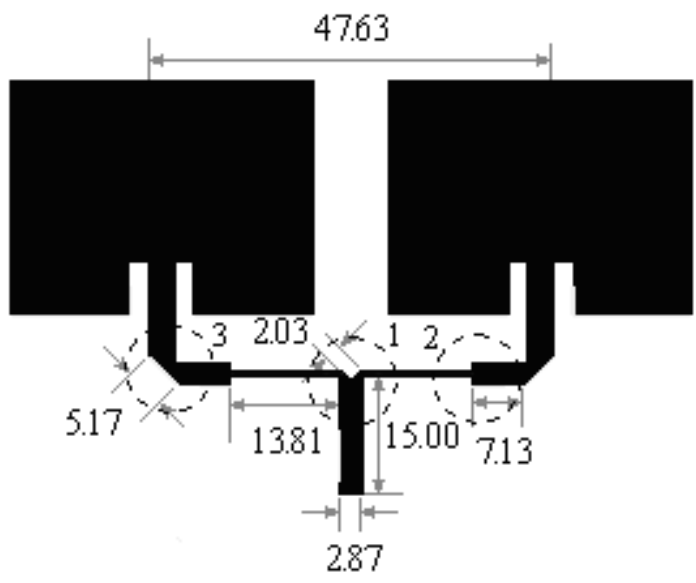

Fig. 2. Proposed array geometry. 
The feed network of the array was designed for the signal can reach the antennas in a same way. For the distribution of the equalized signal to both elements of the array, some discontinuities have been incorporated in feed lines that make up the network. The discontinuity circulated with the number (1) is called T-junction and it is, perhaps, one of the most important discontinuities of devices used in microstrip. The T-junction is designed to divide equally the signal to both elements of the array. The discontinuity (2) known as impedance transformer is intended to obtain an impedance matching of the microstrip lines with different impedances. Finally, the discontinuity (3) called bend is used to introduce flexibility in the layout design of the feed network. The cuts in existing discontinuities (1) and (3) are intended to compensate losses caused by such discontinuities. The dimensions of the feed network were calculated from formulas found in traditional literature [19].

The array was designed to operate in IEEE $802.11 \mathrm{~b} / \mathrm{g} / \mathrm{n}$, at $2.45 \mathrm{GHz}$, with a minimum bandwidth of $83.5 \mathrm{MHz}$ (3.41\% fractional bandwidth), and an input impedance of $50 \Omega$. After designing, the array was simulated with Ansoft HFSS. The parameters obtained were: reflection coefficient $\left(S_{11}\right)$, radiation pattern, gain, input impedance, directivity, surface waves (electric field), half power angle, radiation efficiency and front-to-back ratio. For a frequency of $2.45 \mathrm{GHz}$ the input impedance obtained with the simulation was $50.9 \Omega$ and the bandwidth was $127 \mathrm{MHz}$ (5.18\% fractional bandwidth), which meets the requirements of the desired application. The simulated gain for that frequency was $4.46 \mathrm{dBi}$ and directivity was $7.23 \mathrm{~dB}$.

After simulation, the array was built and $S_{11}$ measurements were performed with a vector network analyzer ZVB-14 model, from Rohde \& Schwarz. As can be seen in Fig. 3, there is a good agreement between measured and simulated results. The measured results show a resonance frequency of 2.53 GHz. The small difference points to an error of $-3.26 \%$.

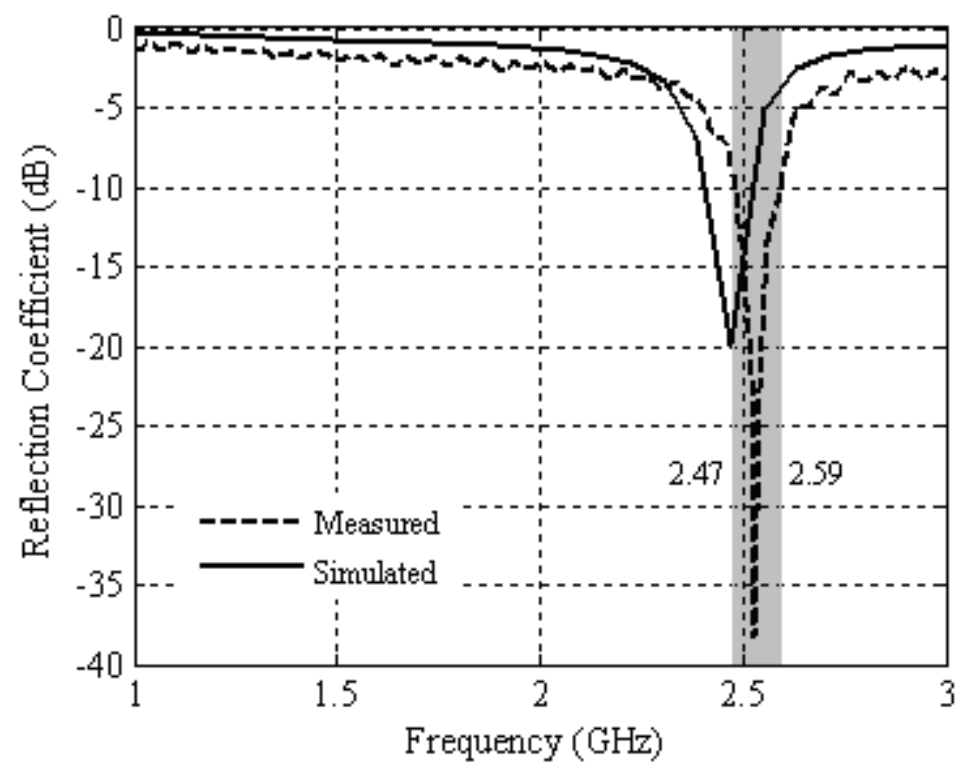

Fig 3. Module of return loss for the conventional array, comparison between numerical and measured results. 
In addition to the simulated results, the radiation patterns in the $\mathrm{E}$ and $\mathrm{H}$ planes are illustrated in Fig. 4 , at the frequency of $2.45 \mathrm{GHz}$. From the radiation pattern some parameters were obtained. The half-power angle for this array was $40^{\circ}$. The radiation efficiency was around $53 \%$ and the front-toback ratio was $14.6 \mathrm{~dB}$.

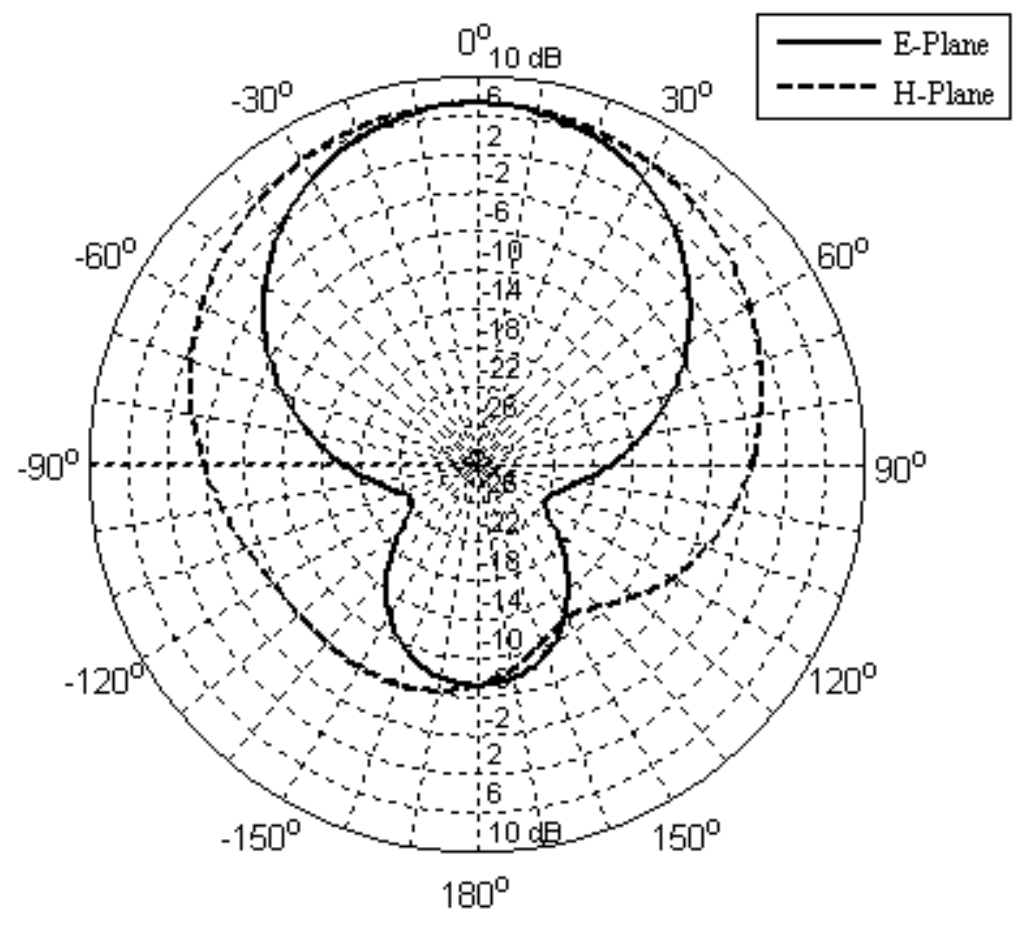

Fig 4. Radiation pattern for the conventional array.

\section{PROPOSED FSS}

We are proposing a new configuration of microstrip antenna array integrated with FSS. This configuration presents improved performance of many parameters. The idea is to employ this new configuration in WLAN and LTE 4G technologies. To improve some parameters such as gain, surface waves, radiation efficiency, directivity and front-to-back ratio, we use a reflector formed by a stopband FSS and a FSS superstrate formed by a band-pass structure.

The proposed FSS are designed to operate in the frequency range $2-3 \mathrm{GHz}$, with resonance frequency at $2.45 \mathrm{GHz}$. The chosen geometry was the square loop element in both FSS. The choice of this geometry is because, besides having square loop stability and polarization angle, we can reduce dimensions at the desired frequency band due to its large electrical length. The material used was the same of the array (FR4). Fig. 5 illustrates the geometry of the FSS used in the study. 


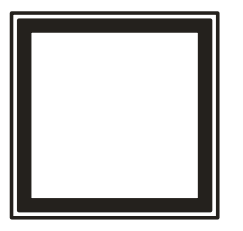

(a)

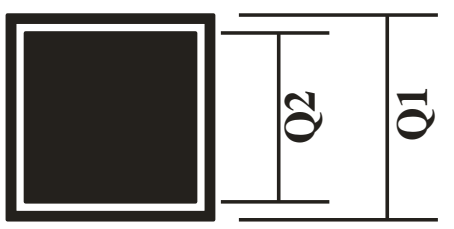

(b)

Fig. 5. Geometry of the FSS element: (a) stop band and (b) band pass.

The dimensions of the designed FSS are as follow: Q1 outer square $(19 \mathrm{~mm} \times 19 \mathrm{~mm})$ inner square Q2 $(16.85 \mathrm{~mm} \times 16.85 \mathrm{~mm})$ and a periodicity of $20 \mathrm{~mm}$ in both $x$ and $y$ directions. The number of the elements used in the FSS was 72 ( 8 rows and 9 columns each).

Based on the resonance frequency curves of the transmission coefficient of the FSS stop-band designed as a function of frequency for angles of incidence between $0^{\circ}-45^{\circ}$ for both wave polarizations (horizontal and vertical) are shown in Fig 6(a) and 6(b).

As can we can see in Fig. 6 the rejection bandwidth is bigger than $2 \mathrm{GHz}(2-3 \mathrm{GHz})$ for various angles of incidence and vertical and horizontal polarization. The results show that for the desired range of operation $(2.4-2.7 \mathrm{GHz})$ the FSS provides angular and polarization stability for a $-10 \mathrm{~dB}$ level. Additionally, the FSS has a linear frequency response for the angle of the reflection coefficient within the desired bandwidth, which can be very useful in pulsed systems, in which a linear phase may be a prerequisite.

Fig. 7(a) and 7(b) illustrate the bandwidths of transmission coefficient for the band-pass FSS for horizontal and vertical polarizations, respectively, and for several angles of incidence. It can be seen that the FSS has an insertion loss less than $0.5 \mathrm{~dB}$ in the desired range of operation $(2.4-2.7 \mathrm{GHz})$. As the stop-band FSS, the band-pass structure also presents angular and polarization stability, and a linear frequency response for the angle of the reflection coefficient in the desired range of frequency. 


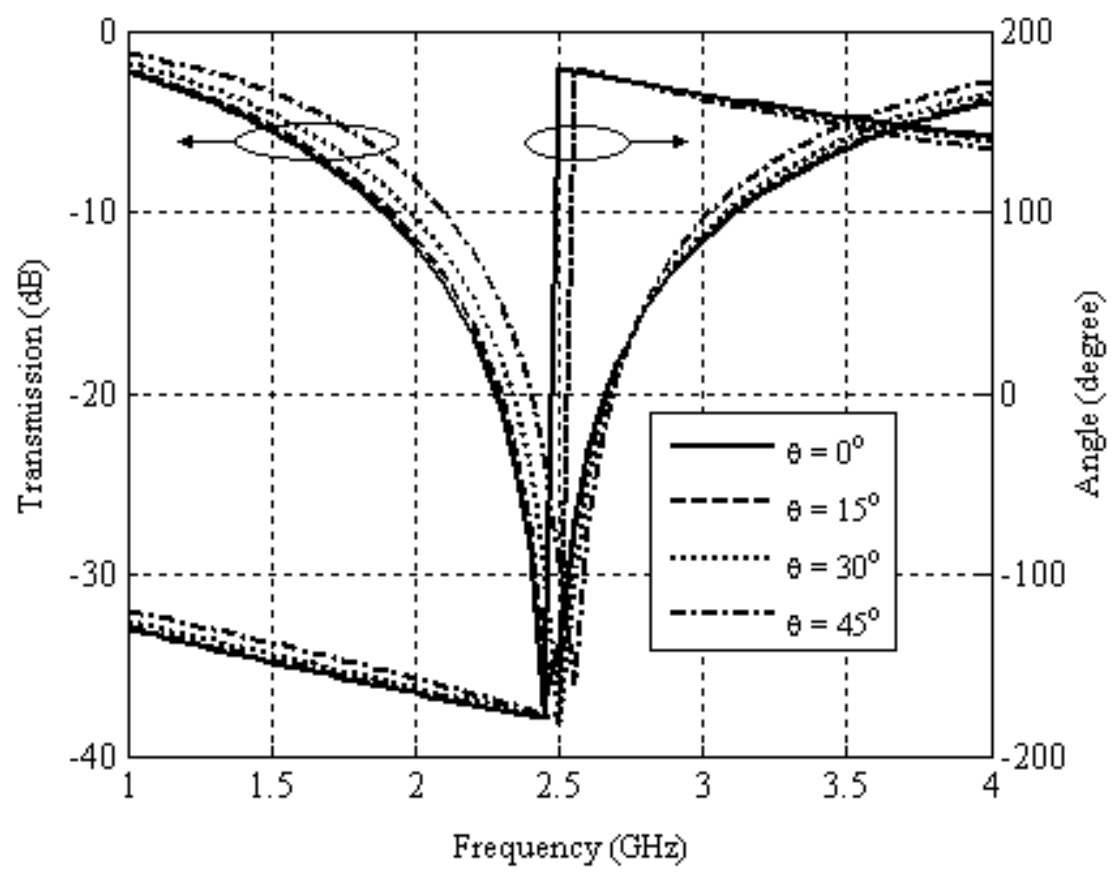

(a)

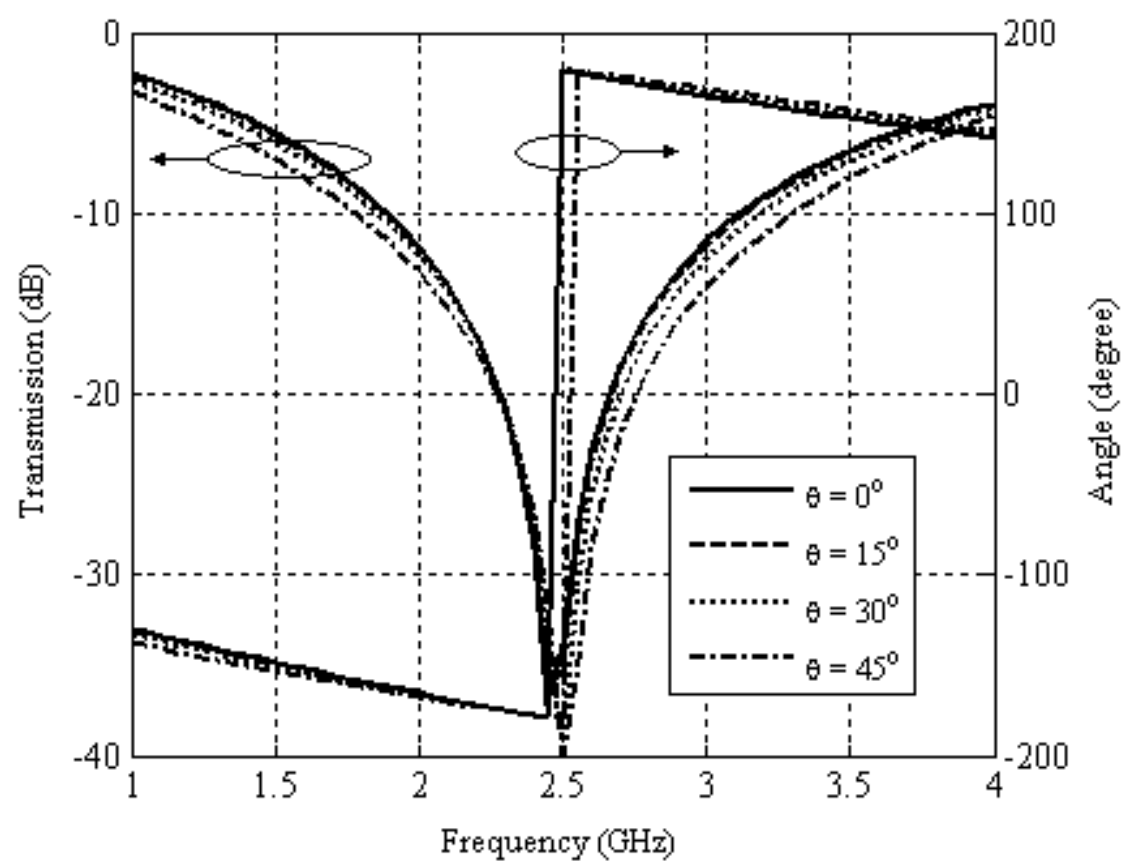

(b)

Fig. 6. Transmission and phase of the reflection coefficient versus frequency for FSS stop-band: (a) horizontal polarization and (b) vertical polarization. 


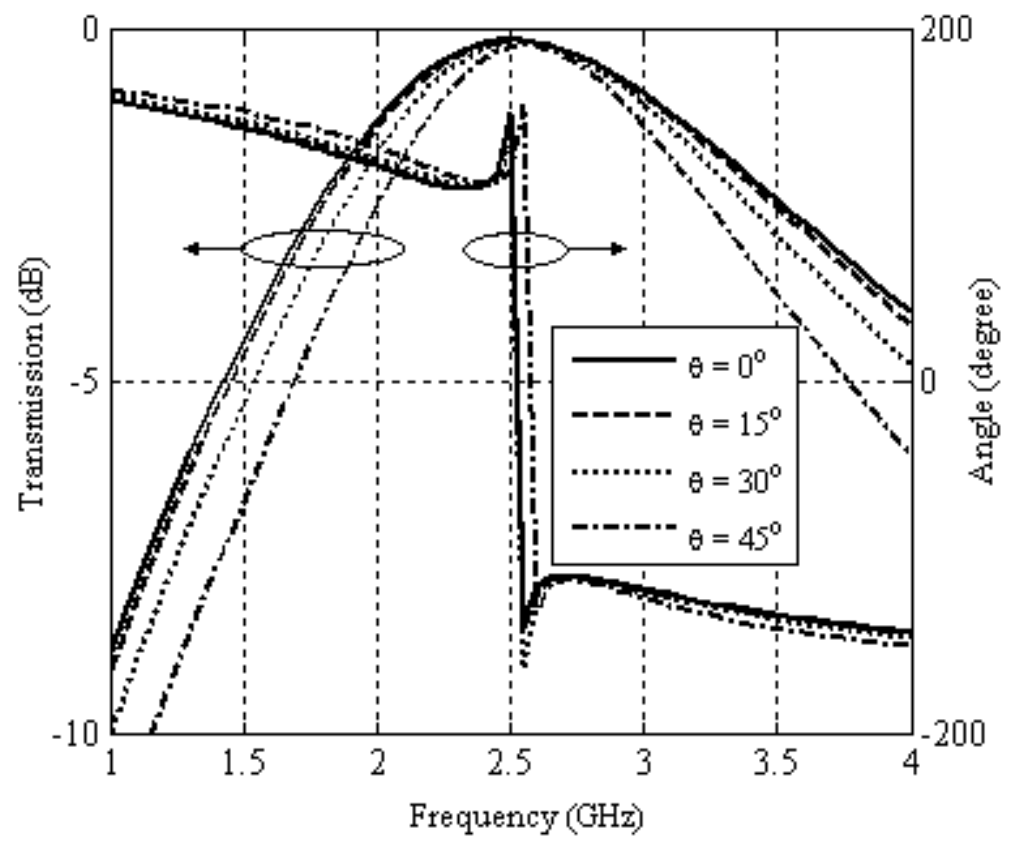

(a)

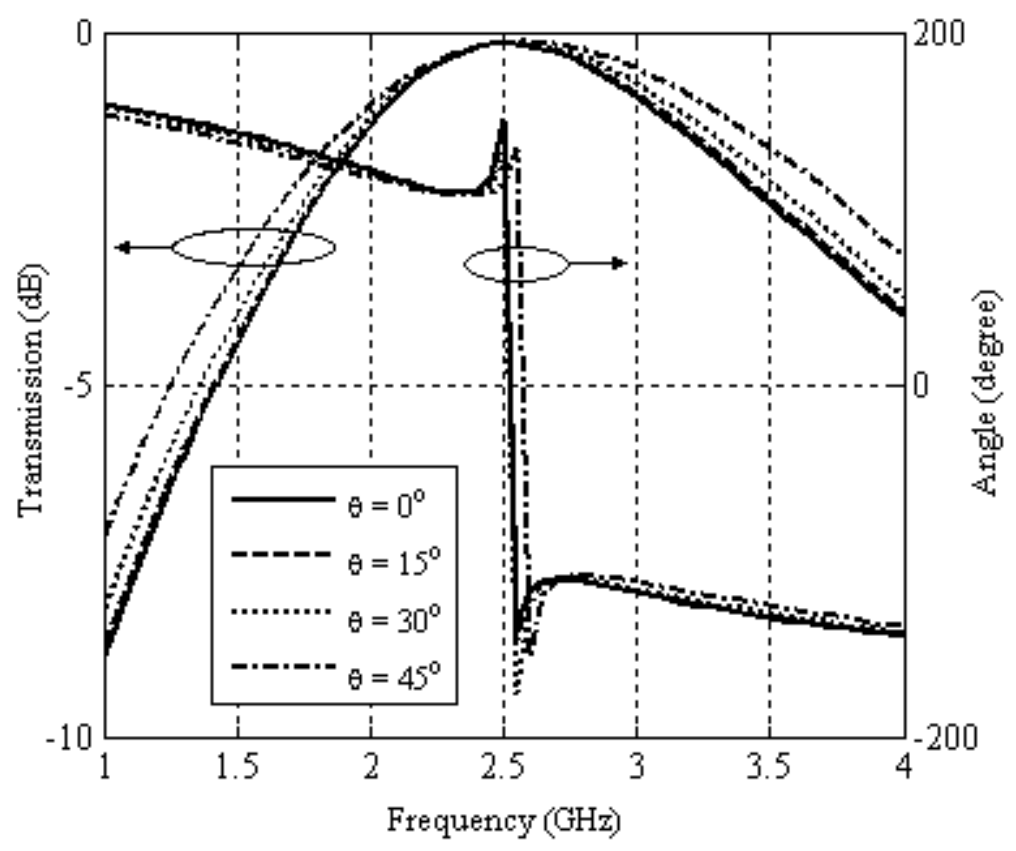

(b)

Fig. 7. Transmission and phase of the reflection coefficient versus frequency for FSS pass-band: (a) horizontal polarization and (b) vertical polarization.

\section{PROPOSED ARRAY WITH OPTIMIZED PARAMETERS}

As stated previously, the integration of an array of microstrip antennas with FSS proposes to improve the performance of the main radiation parameters of the array, such as gain, directivity, frontback ratio and reduced surface waves. Other parameters that can be optimized are: bandwidth and the 
array size. One form found in literature to increase the bandwidth of a microstrip antenna array is truncating the ground plane [20]. Analyzing the results obtained for the array with full ground plane, it is observed that the fractional bandwidth of the array is narrow (5.18\%). To try to overlap this problem, we propose the truncation of the array's ground plane. This configuration is shown in Fig. 8 .

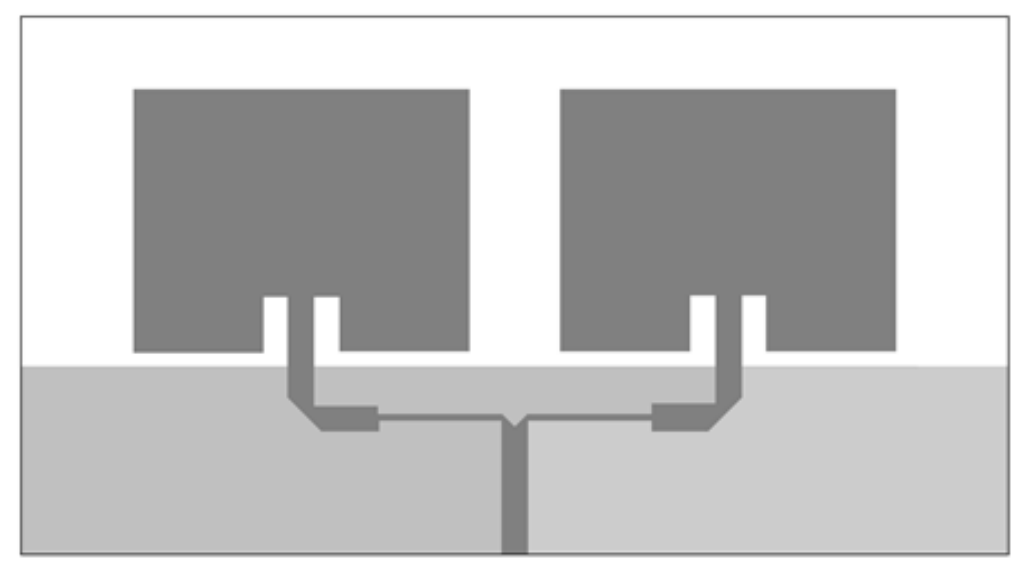

Fig. 8. Array with truncated ground plane.

Reducing the ground plane of the array, we observed an increase in the bandwidth as well as a reduction in the resonant frequency. The resonant frequency for the new configuration was $1.76 \mathrm{GHz}$ with a bandwidth of $521 \mathrm{MHz}$ (29.6\% fractional bandwidth). This reduction in resonant frequency shows that we can reduce the size of the elements of the array so that the resonant frequency back to the desired value $(2.45 \mathrm{GHz})$. Thus, the dimensions of the elements of the array were reduced by $40 \%$. Before that, the resonance frequency was equal proximately to $2.45 \mathrm{GHz}(2.52 \mathrm{GHz})$ and bandwidth (465 MHz) was close to the bandwidth of the array with truncated ground plane (521 MHz), resulting in a fractional bandwidth of $18.41 \%$.

However, truncating the ground plane the array ceases to be directive and will operate as omnidirectional. So, the simulated gain for frequency of $2.52 \mathrm{GHz}$ was $0.76 \mathrm{dBi}$ and directivity was $1.28 \mathrm{~dB}$, the half power angle of $90^{\circ}$ and a front-to-back ratio was $0.082 \mathrm{~dB}$. Although there has been a considerable reduction in the gain and directivity of the array with truncated and reduced ground plane, there was also a significant improvement in bandwidth. To try to solve the problem of reducing the gain and directivity of the array with truncated ground plane, we put below of the array a stopband FSS (such a ground plane). This configuration can be seen in Fig. 9.

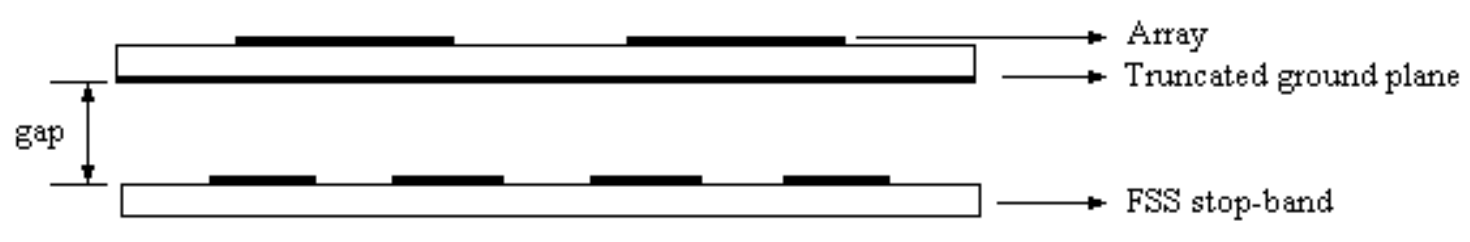

Fig. 9. Array with truncated ground plane and stop band FSS. 
For this configuration the gap was varied to obtain the optimum distance between the array and FSS. The best gap was $3 \mathrm{~cm}$. Thus, the gain was increased to $5.70 \mathrm{dBi}$ and directivity to $6.20 \mathrm{~dB}$, halfpower angle was $60^{\circ}$, the front-to-back ratio was $12.83 \mathrm{~dB}$ and the bandwidth increased to $762 \mathrm{MHz}$. It shows a significant improvement in the parameters of the array.

Trying to obtain a result even more significant, we used a third layout: The antenna array with truncated ground plane, integrated with a stop-band FSS as ground plane and a band-pass FSS as superstrate. The idea is to reduce the surface waves and further improve the parameters of the array. Fig. 10 illustrates this configuration.

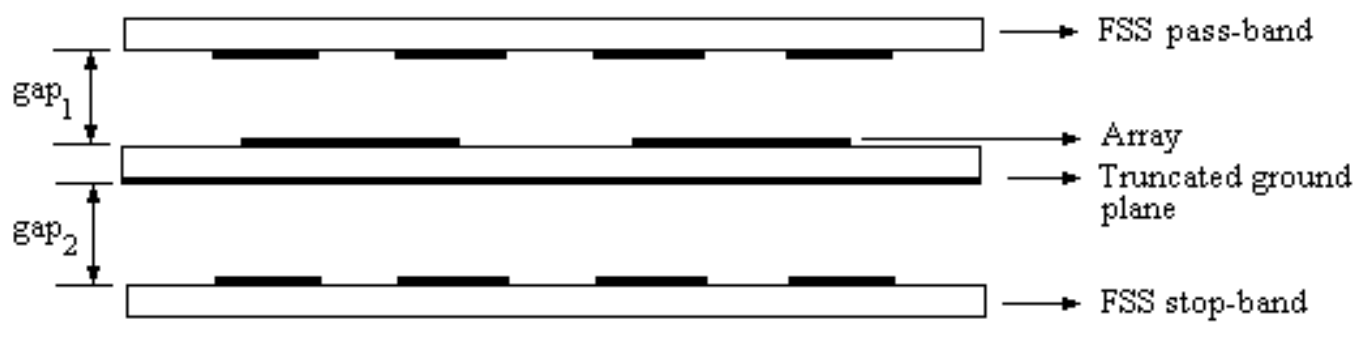

Fig. 10. Array with truncated ground plane integrated with stop band FSS, as ground plane, and with pass band FSS, as superstrate.

The best values for the gaps 1 and 2 were $1 \mathrm{~cm}$ and $3 \mathrm{~cm}$, respectively. For this configuration, the bandwidth decreased to $672 \mathrm{MHz}$, at $2.45 \mathrm{GHz}$ frequency the gain has been increased to $7.26 \mathrm{dBi}$, a half-power angle of $60^{\circ}$ was kept in, the front-to-back ratio was increased to $17.33 \mathrm{~dB}$ and radiation efficiency was around $85 \%$. For the $2.60 \mathrm{GHz}$ frequency the gain was $7.67 \mathrm{dBi}$, the half power angle of $60^{\circ}$ was kept in, the front-to-back ratio was $16.81 \mathrm{~dB}$ and radiation efficiency was around $80 \%$. It can be observed that the new configuration parameters remained close for the two frequency bands of desired applications. The reduction in efficiency is due to a not flat response of the FSS superstrate, causing a larger insertion loss for the frequency of $2.60 \mathrm{GHz}$. Still, it can be seen that there was an improvement in the parameters of the previous configuration, although small decrease in bandwidth. Fig. 11 illustrates the reflection coefficient for this layout, compared with the other configurations.

The significant improvement in bandwidth allows this new array to be employed in two applications, which are: WLAN and LTE 4G networks. The radiation diagrams in the E and H planes to the center frequencies of these two applications are illustrated in Fig. 12(a) and 12(b), these results were obtained using Ansoft HFSS. From the radiation pattern of some parameters, for both frequencies were obtained. 


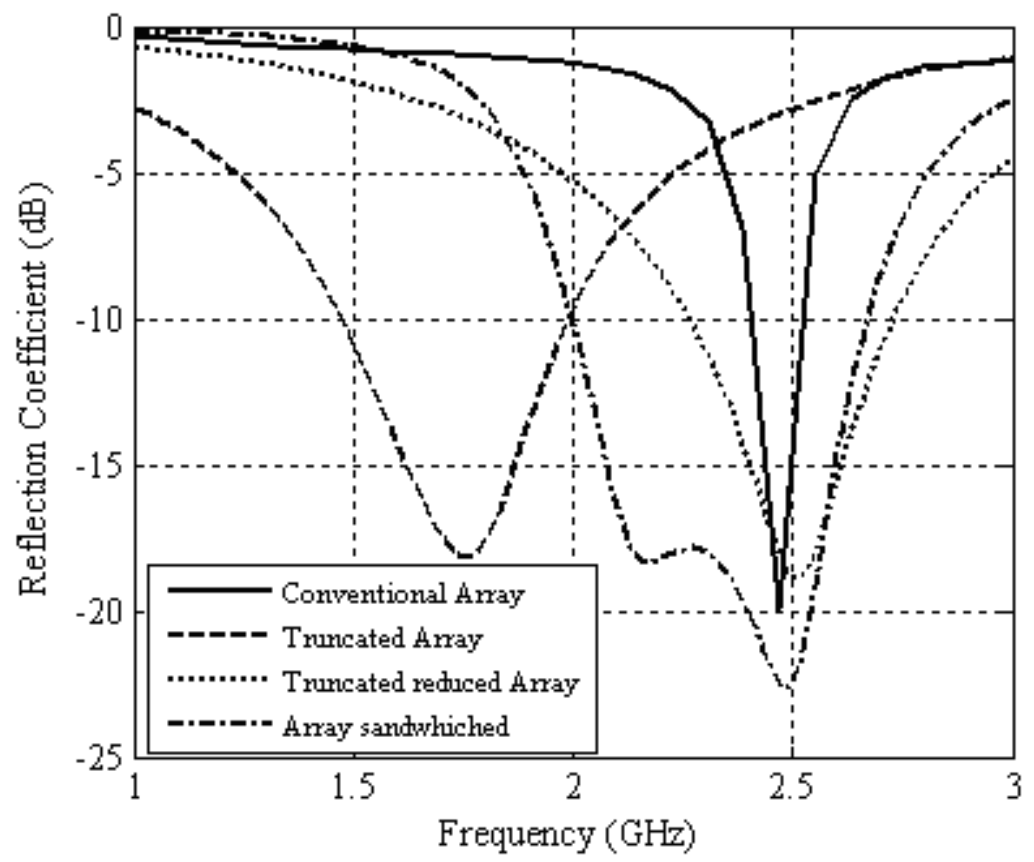

Fig. 11. Comparison of the reflection coefficient obtained for all proposed layouts.

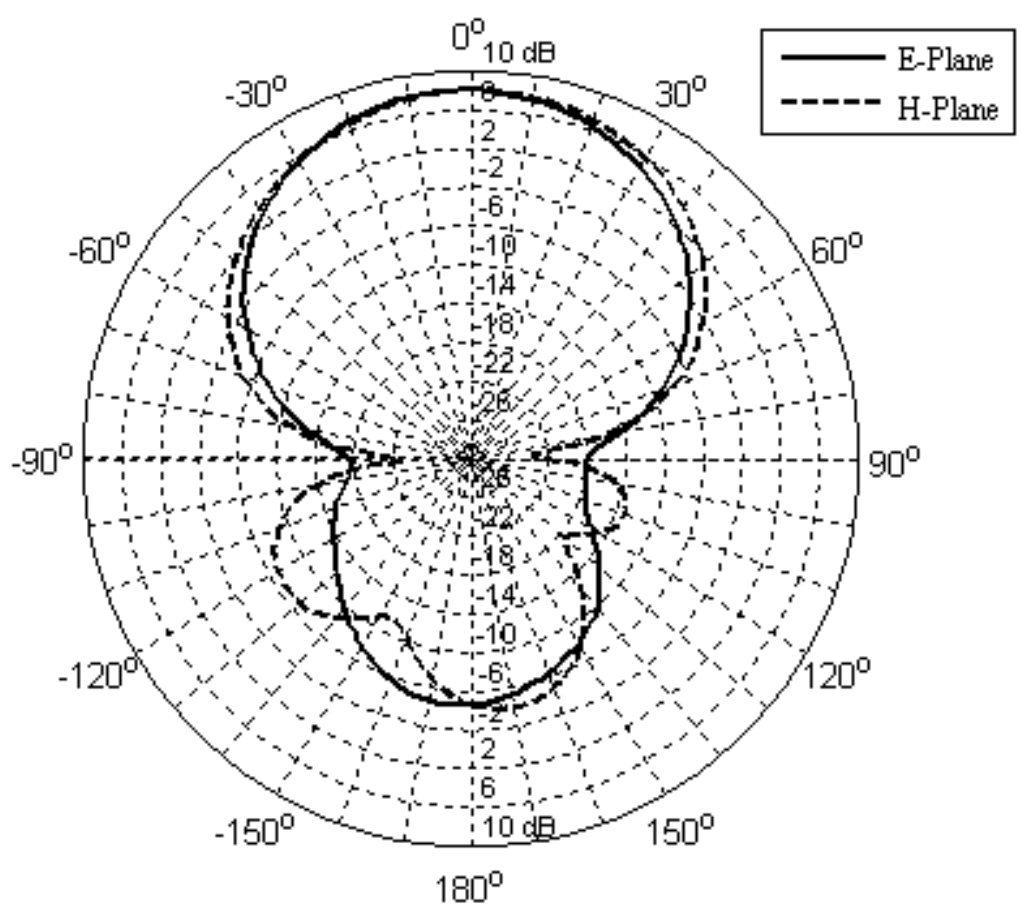

(a) 


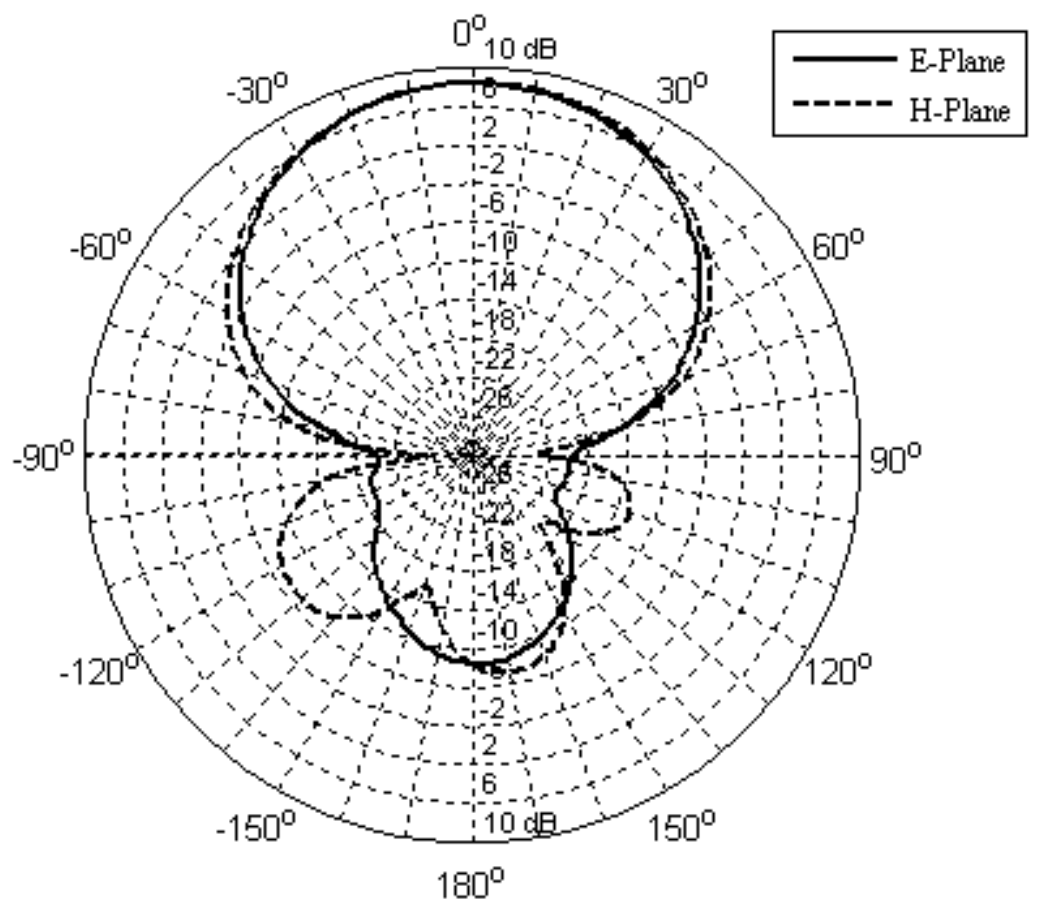

(b)

Fig. 12. Radiation patterns for: (a) 2,45 GHz and (b) 2,60 GHz.

To illustrate how the band-pass FSS, used as a superstrate influences the array's surface waves distribution, graphs of the surface waves were obtained of all settings for the frequency of $2.45 \mathrm{GHz}$ Fig, 13 illustrates the electric field distribution for the frequency $2.45 \mathrm{GHz}$ in case of the conventional array. We can observe an intense electric field in the region of the dielectric, which decreases the radiation efficiency of the array. For this case, the efficiency radiation is $52.8 \%$. So, we can observe high intensities of surface waves.

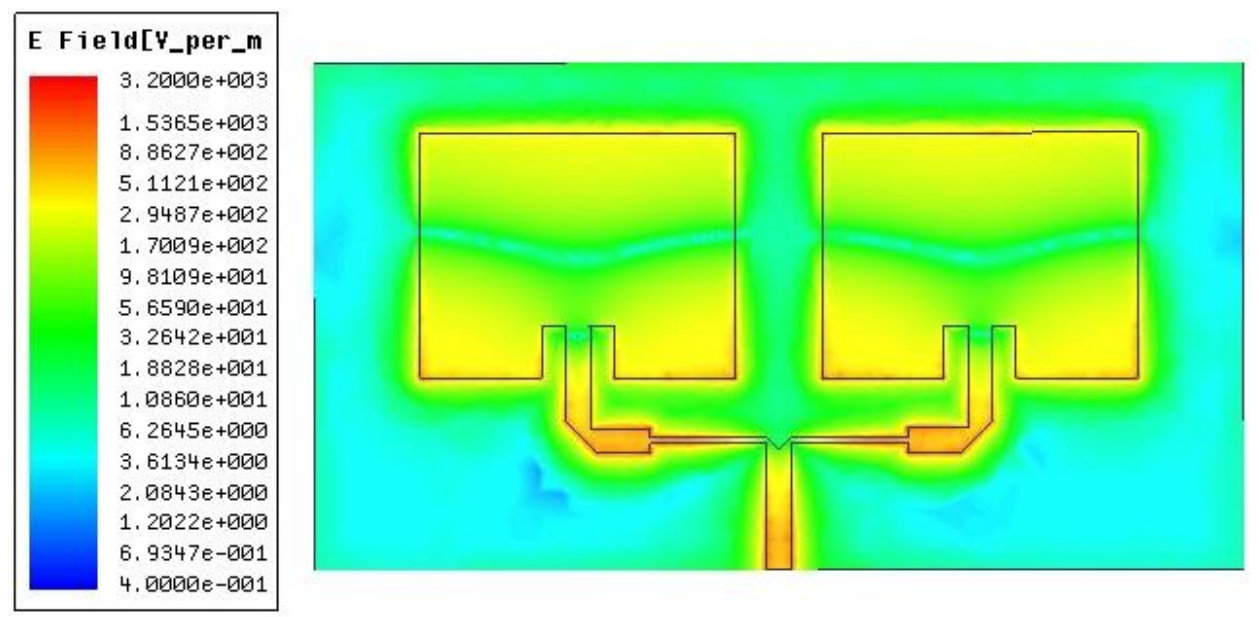

Fig. 13. Electric field distribution for the conventional array. 
For the sandwiched array, it is observed that there is a visible reduction in the concentration of the electric field in the vicinity of the array elements. This entails a greater insulation between the elements of the array, as well as greater efficiency of radiation. Fig. 14(a) and 14(b) illustrate the surface waves of the electric field at frequencies of $2.45 \mathrm{GHz}$ and $2.60 \mathrm{GHz}$, respectively, for the case of the array with truncated ground plane between two FSS. It can be observed a significant reduction in surface waves, compared with the previous case.

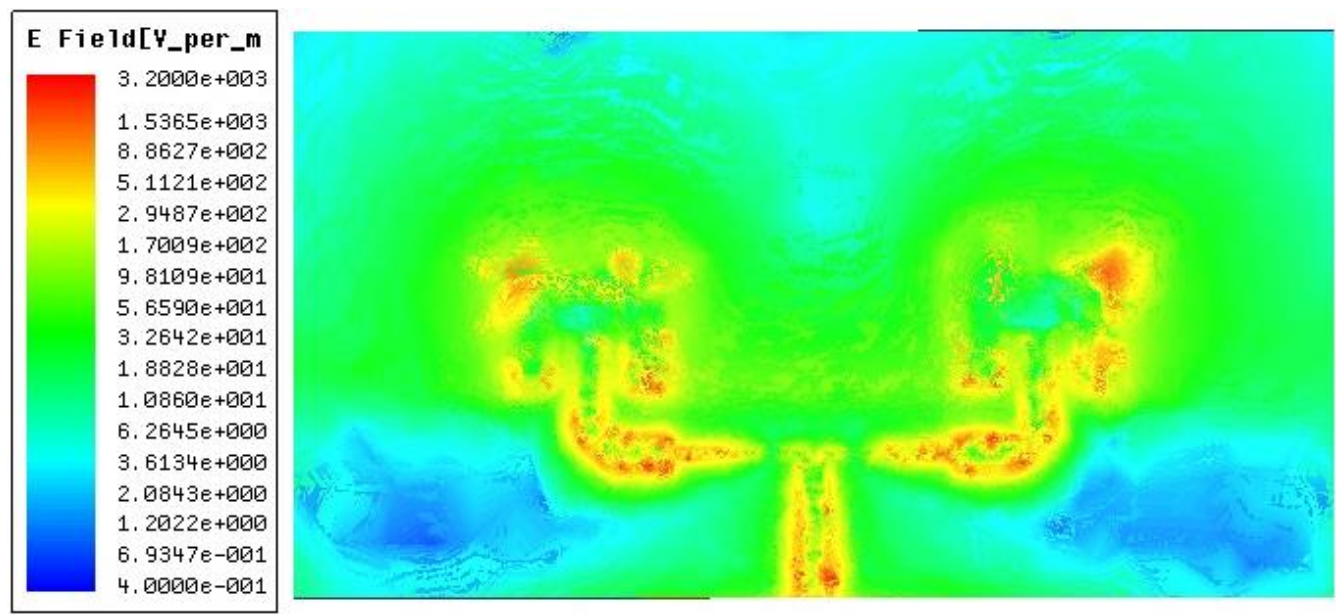

(a)

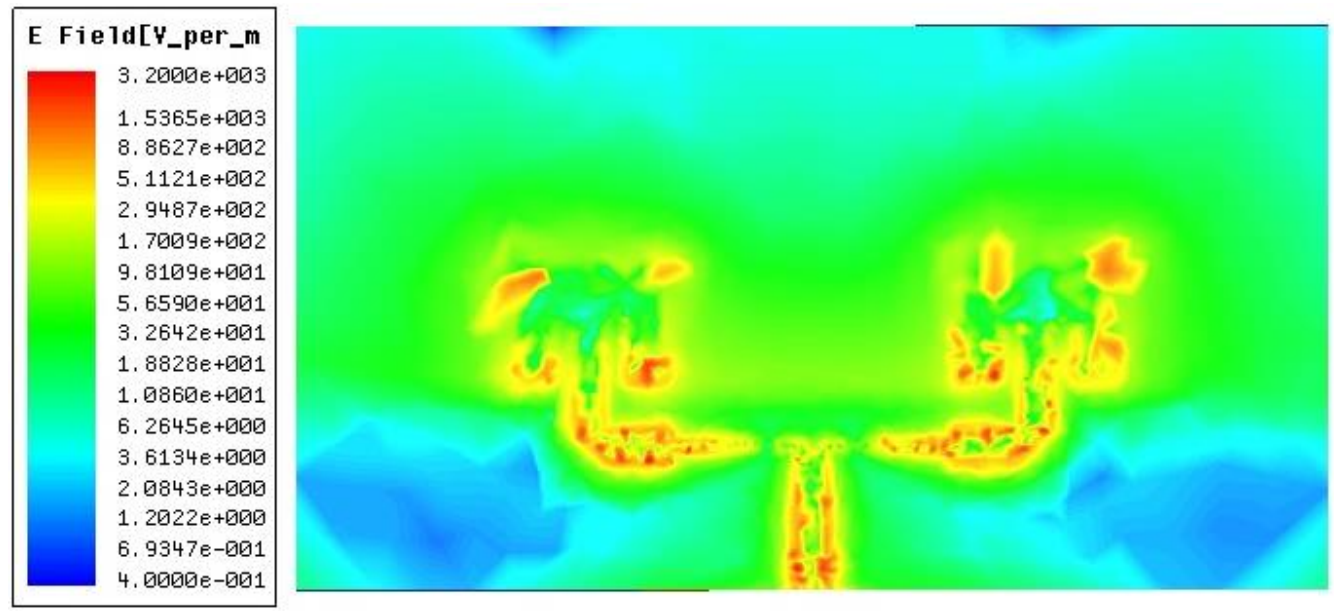

(b)

Fig. 14. Electric field distribution for the array with truncated ground plane between two FSS, at frequency of: (a) $2.45 \mathrm{GHz}$ and (b) $2.60 \mathrm{GHz}$.

To confirm the analysis developed the two FSS and the array with truncated ground plane were built. Measurements of the $S_{11}$ were performed. Fig. 15 illustrates the measurement setup and how the FSS were integrated with the array. Fig. 16 illustrates the comparison between simulated and measured results. We can observe a good agreement 
between the results and we show that the array between FSS can be used in the deried applications (WLAN and 4G).

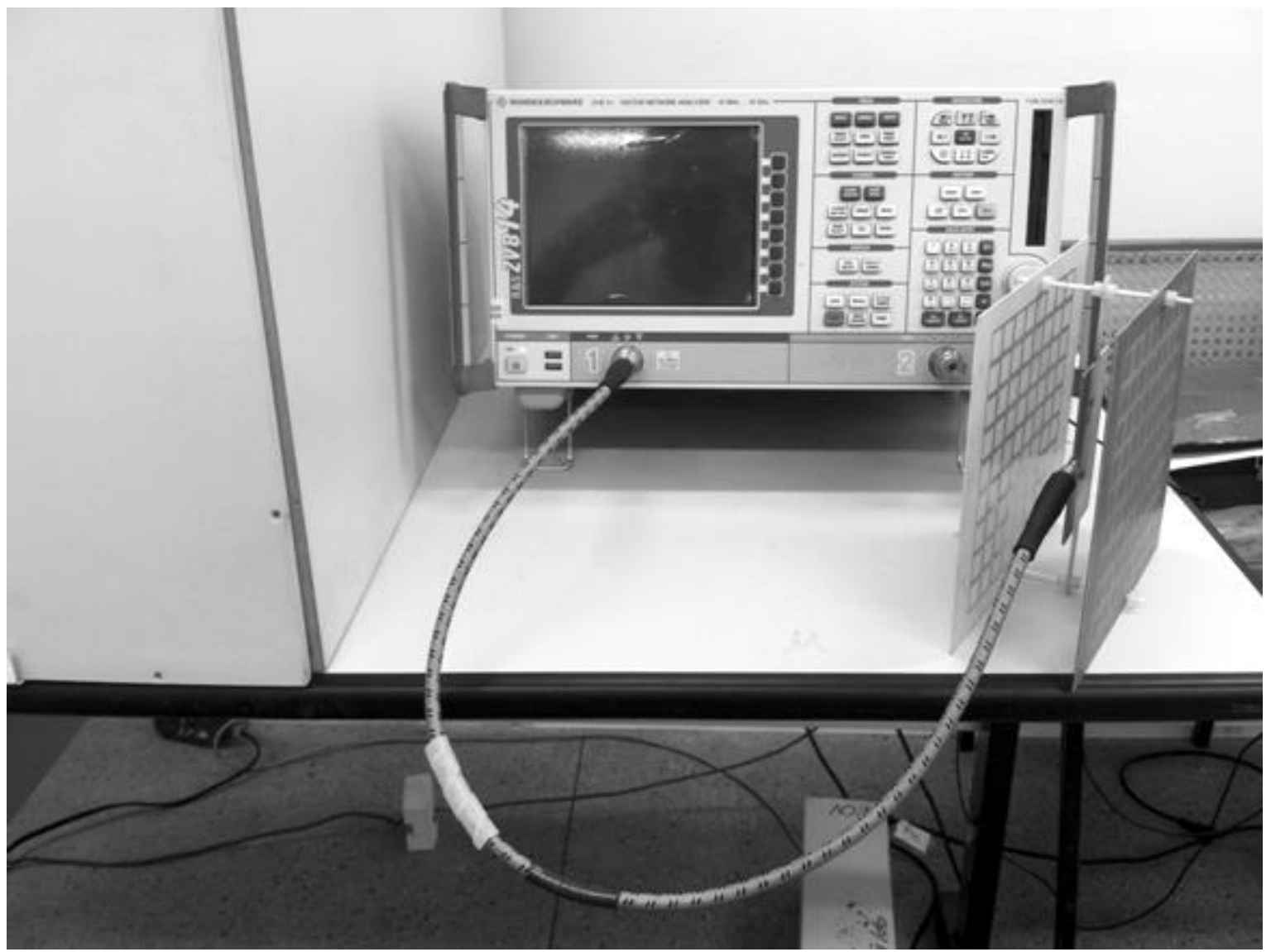

Fig. 15. Measurement setup and built prototypes.

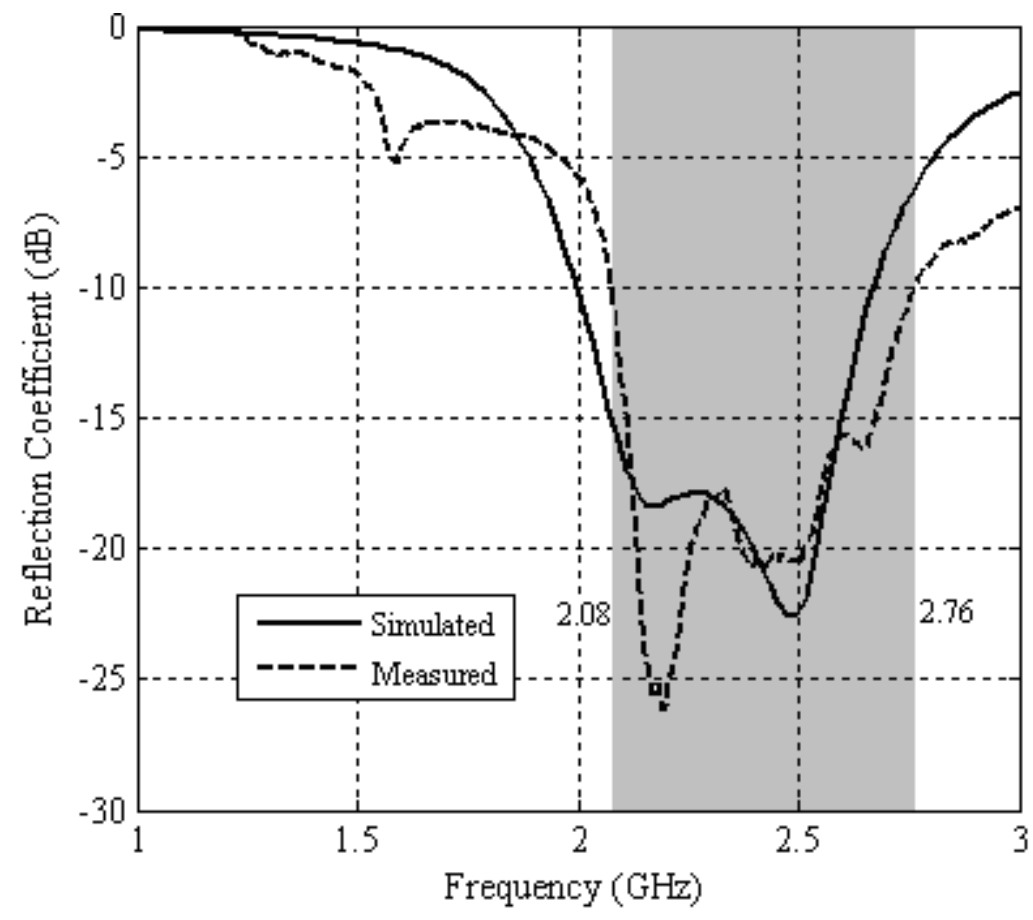

Fig. 16. Comparison between simulated and measured results for $S_{11}$. 
At the end of the study, the optimal array obtained is illustrated in Fig. 17. We can observe a reduction in the dimensions of the patches which leads to a reduction in the dimensions of the array as a whole.

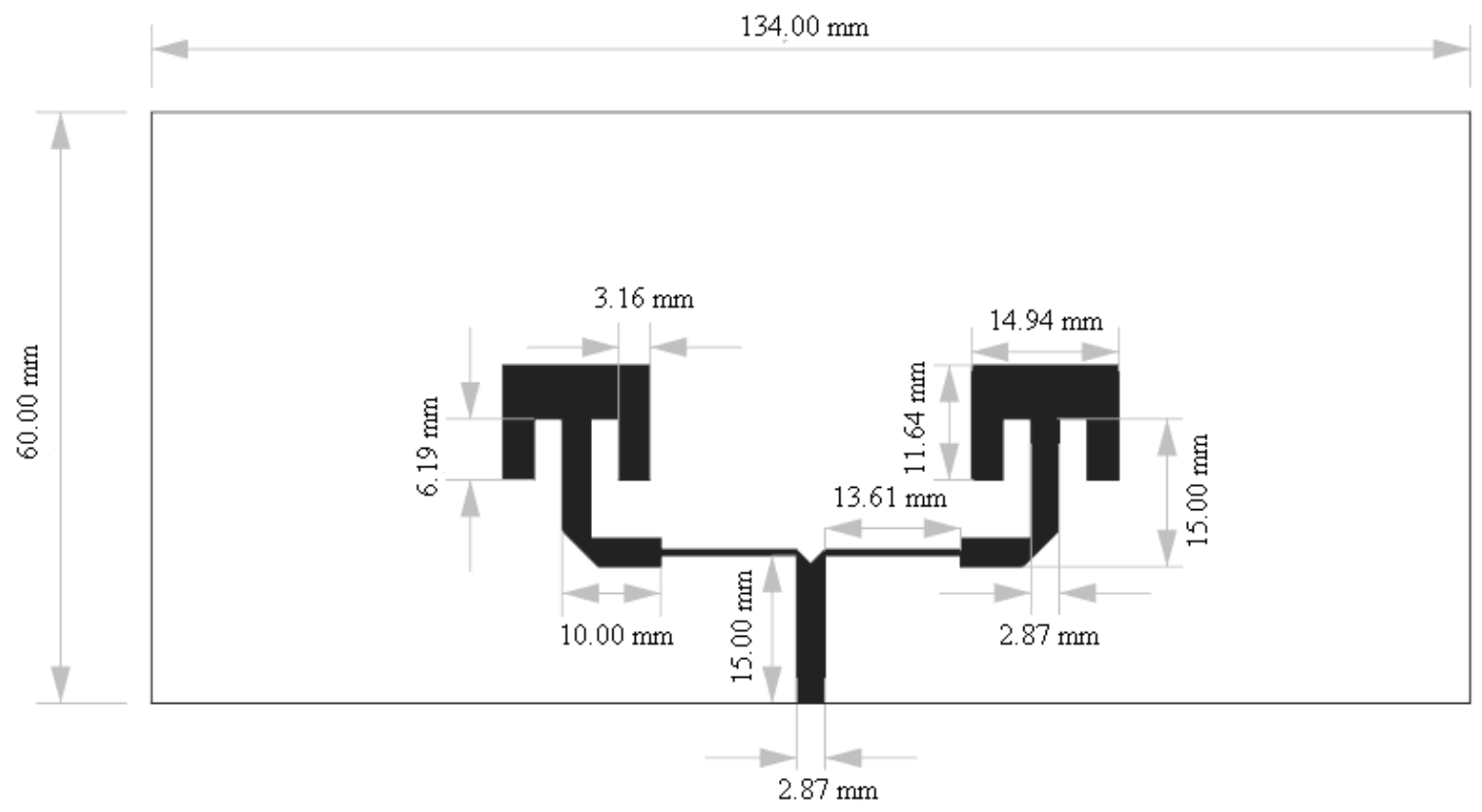

(a)

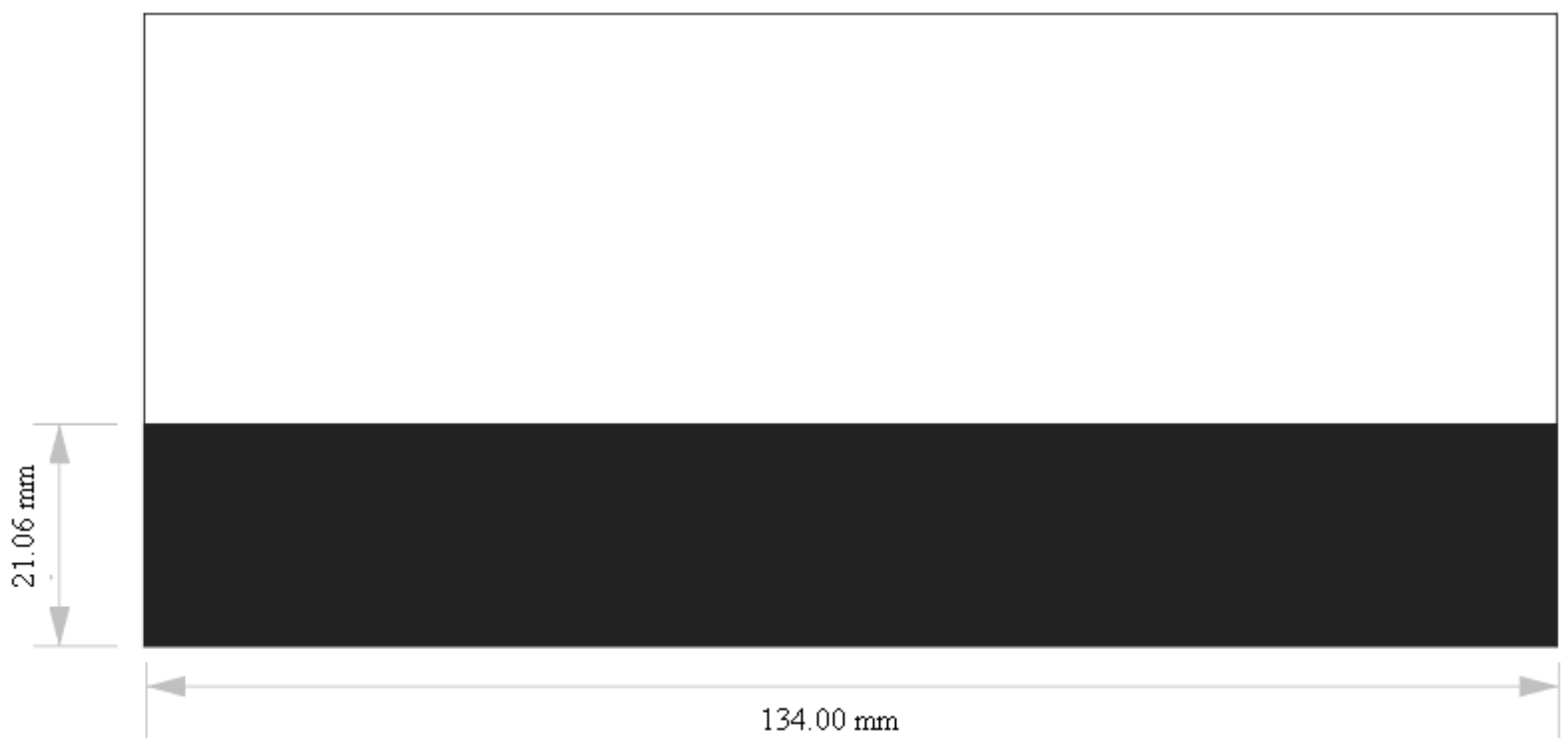

(b)

Fig. 17. Optimal array: (a) Front view and (b) Back view. 


\section{CONCLUSIONS}

The application of frequency selective surfaces in the antenna arrays proved to be a good alternative when you want to modify radiation parameters of the array. It was found that there is a better distance, for which this interaction between the FSS and the array provides the best results. In this case, the values were $1 \mathrm{~cm}$ and $3 \mathrm{~cm}$ for the FSS superstrate and ground plane, respectively. The parameters of the original array have been significantly improved, in particular the bandwidth, gain and radiation efficiency. The new proposed configuration can be used in panel directivity antennas for systems with central base station sectorized cells in commercial applications of WLAN and $4 \mathrm{G}$.

\section{REFERENCES}

[1] Islam, M. M., Islam, M. T., and Faruque, M. R. I., "Dual-Band Operation of a Microstrip Patch Antenna on a Duroid 5870 Substrate for Ku- and K-Bands," The Scientific World Journal, pp. 1-10, 2013.

[2] Samsuzzaman, M., Islam, M. T., and Faruque, M. R. I., "Dual-band multi slot patch antenna for wireless applications," Journal of Telecommunications and Information Technology, vol. 2, pp. 19-23, 2013.

[3] Godara, L. C., Handbook of Antennas in Wireless Communications, CRC Press, New York, 2002.

[4] Huang, Y. and Boyle, K., Antennas From Theory to Practice, John Wiley \& Sons, New Jersey, 2008.

[5] Subbulakshmi, P. and Rajkumar, R., "Design and characterization of corporate feed rectangular microstrip patch array antenna", International Conference on Emerging Trends in Computing, Communication and Nanotechnology (ICE-CCN), pp. 547 - 552, 2013.

[6] Wolansky, D., Vsetula, P., Puskely, J. and Raida, Z., "Broadband small patch antenna array for Ka-band application", 7th European Conference on Antennas and Propagation (EuCAP), pp. 907 - 910, 2013.

[7] Hamoudi, H., Haddad, B. and Lognonne, P., "Study of a L2 patch antennas arrays for GNSS/GPS network", 7th European Conference on Antennas and Propagation (EuCAP), pp. 2187 - 2191, 2013.

[8] Gruden, M., Jobs, M. and Rydberg, A., "Design and evaluation of conformal patch antenna array use with wireless sensor network inside jet engines", 7th European Conference on Antennas and Propagation (EuCAP), pp. 2100 - 2102, 2013.

[9] Chahat, N., Zhadobov, M., Muhammad, S. A., Le Coq, L. and Sauleau, R., "60-GHz Textile Antenna Array for Body-Centric Communications", IEEE Transactions on Antennas and Propagation, Vol. 61, pp. 1816 - 1824, 2013.

[10] Anguera, J., Montesinos, G., Puente, C., Borja, C., Soler, J. "An under-sampled high directivity microstrip patch array with a reduced number of radiating elements inspired on the Sierpinski fractal”, Microwave and Optical Technology Letters, Vol. 37, No. 2, pp. 100$103,2003$.

[11] Jayasinghe, J. M. J. W., Anguera, J. and Uduwawala, D. N., "Genetic algorithm optimization of a high-directivity microstrip patch antenna having a rectangular profile", Radioengineering, Vol.22, No. 3, pp. $700-707,2013$.

[12] Kim, D., J. Yeo and J. Choi (2008), 'Compact spatial triple-band-stop filter for cellular/PCS/IMT-2000 systems', ETRI Journal.

[13] Chaimool, S., Rakluea, C., and Akkaraekthalin, P., "Compact wideband microstrip thinned array antenna using EBG superstrate", International Journal of Electronics and Communications (AEÜ), no. 66, pp. 49 - 56, 2012.

[14] Chen, Hsing-Yi (2010), 'Bandwidth enhancement using dual-band frequency selective surface with Jerusalem cross elements for 2.4/5.8 GHz WLAN antennas', IEEE International Conference on Wireless Information Technology and Systems, pp. 1-4.

[15] Yongxing, Che (2010), "Design of multiple FSS screens with dissimilar periodicities for directivity enhancement of a dual-band patch antenna", International Symposium on Antennas Propagation and EM Theory, pp. 319-322.

[16] Ranga, Y., L. Matekovits, K. P. Esselle and A. R. Weily, "Enhanced gain UWB slot antenna with multilayer frequency selective surface reflector", International Workshop on Antenna Technology, pp. 176-179, 2011.

[17] Wang, Y., Huang, J. and Feng, Z., "A Novel Fractal MultiOBand EBG Structure and Its Application in MultiOAntennas," IEEE [18] Antennas and Propagation Society, International Symposium, pp. 5447 0 5450, 2007.

[19] Visser, H. J. (2005), Array and Phased Array Antenna Basics, John Wiley \& Sons Ltd, England.

[20] Huang, J., "The finite ground plane effect on the microstrip antena radiation patterns", IEEE Transactions on Antennas and Propagation, pp. 649-653, 1983. 